Gdańsk 2019, Nr. 41

https://doi.org/10.26881/sgg.2019.41.23

Magdalena Filar

Jagiellonen-Universität in Krakau

https://orcid.org/0000-0002-6078-9758

\title{
Stereotype von Polen im deutschen Weltbild des 19. Jahrhunderts. Eine ethnolinguistische Studie
}

Der Beitrag hat zum Ziel, die ethnolinguistische Konzeption der Untersuchung von Stereotypen von Jerzy Bartmiński vor dem Hintergrund anderer kognitiver Ansätze zu diskutieren und die Stereotype von Polen im deutschen Weltbild des 19. Jahrhunderts zu erforschen. Die ethnolinguistische Analyse konzentriert sich auf die Rekonstruktion der Bedeutung von Stereotypen über Polen und auf die Bestimmung von deren Profilen auf der textuellen Ebene sowie auf die Beschreibung des Stereotypenwandels, der sich im Laufe des 19. Jahrhunderts vollzogen hat. Das Material der Analyse bilden Phraseologismen und Metaphern, die lexikographischen Quellen und den Texten des DWDS Korpus (Textarchiv 1800-1900) entnommen wurden.

Schlüsselwörter: die kognitive Definition, Profilierung, Profil, nationale Stereotype, der Standpunkt

Stereotypes about Poland in the German Worldview in the $19^{\text {th }}$ Century. An Ethnolinguistic Analysis. - The aim of this paper is to discuss the ethnolinguistic approach to stereotypes research proposed by Jerzy Bartmiński as compared to other cognitive theories and to analyse the stereotypes about Poland, and the Poles in the German worldview in the $19^{\text {th }}$ century. The ethnolinguistic analysis focuses mainly on the reconstruction of the meaning of the stereotypes about Poland, and the Poles as well as on the determining their most typical profiles, and on the description of the change in these stereotypes throughout the $19^{\text {th }}$ century. The material of the analysis consists of idiomatic expressions and metaphors taken from lexicographical resources and from DWDS Corpus (Textarchiv 1800-1900).

Keywords: cognitive definition, profiling, profile, national stereotypes, point of view

\section{Einleitung}

Die ersten Definitionen des Stereotyps von Lippmann (1922) und Putnam (1975) stellen den Ausgangspunkt für die kognitionslinguistischen Überlegungen über das Phänomen der Stereotypie dar. Unter den vielen kognitiven Ansätzen, die die Stereotype nicht nur als ein psycho- und soziologisches Phänomen, sondern auch als Wissensstrukturen verstehen und diese im Zusammenhang mit Prototypen erwägen (Schemata in der kognitiven Grammatik von LANGACKer 1987, Framesemantik von FILlmore 1975, BusSe 2012, conceptual maps von STEFANOWITSCH 2006), möchte ich auf die Erkenntnisse der kognitiven Ethnolinguistik 
verweisen, die nicht nur in slavistischen (BARTMIŃSKI 1998, 2007, 2012), sondern auch in germanistischen Untersuchungen von Stereotypen Anwendung finden (ZINKEN 2004).

Das Ziel meines Beitrags ist es, Stereotype, ihre Bedeutung und Struktur aus der Sicht der in diesem Ansatz entwickelten Konzeption des sprachlichen Weltbildes zu untersuchen. Ich konzentriere mich dabei (i) auf die Rekonstruktion der Bedeutung von nationalen Stereotypen, sowie (ii) auf die Analyse von deren sprachlichen Ausdrucksmitteln auf der Textebene, darunter insbesondere auf die Analyse von Phraseologismen und Metaphern. Den Hauptgegenstand der Analyse stellen die deutschen Stereotype über Polen im 19. Jahrhundert dar, die vor dem Hintergrund von anderen nationalen Stereotypen dieser Zeit erwogen werden. Das Material der durchgeführten Studie bilden zwei lexikographische Quellen (DWDS Wörterbuch, Online-Lexikon der diachronen Phraseologie) und die Texte des DWDS Textarchivs. ${ }^{1}$

\section{Stereotype und Stereotypenprofile in der kognitiven Ethnolinguistik}

Den theoretischen Rahmen für die Untersuchung der Stereotypie in der Ethnolinguistik stellt der Begriff des ,sprachlichen Weltbildes (auf Polnisch: ,językowy obraz świata', JOS) dar, der in seinen Ursprüngen an die linguistische Idee der „Weltansicht“ (НumboLDT 1836), an die Ansätze von Weisgerber (1971), an die in der Rhetorik postulierten Topoi als Denkmuster sowie an die Konzeption des „naiven Weltbildes“ (Apresjan 1994) anknüpft. Der Begriff des sprachlichen Weltbildes ist nicht nur als ein sprachlich-mentales Bild (LANGACKer 2009), sondern auch als eine „kulturelle Konzeptualisierung“ (CZACHUR 2016) zu verstehen.

BARTMIŃSKI (2012: 266) definiert das sprachliche Weltbild (SWB) ${ }^{2}$ als eine in der Sprache enthaltene Wirklichkeitsinterpretation, die sich als eine Menge von Denkmustern über die Welt, Menschen, Gegenstände, Ereignisse erfassen lässt. Sie ist einerseits subjektiv - es handelt sich dabei um ein aus der Sichtweise eines durchschnittlichen Benutzers gefestigtes Weltbild -, andererseits ist sie auch intersubjektiv, weil sie „der Vergesellschaftlichung unterliegt und zum Bindeglied für die Mitglieder einer Sprach- und Kulturgemeinschaft wird“(ebd). Dementsprechend kann von dem polnischen oder dem deutschen Weltbild gesprochen werden. Der Schwerpunkt der ethnolinguistischen Forschung wird insbesondere auf Stereotype und Wertung gelegt.

BARTMIŃSki $(1998,2007)$ bezieht sich in seiner Auffassung der Stereotype vor allem auf Putnams Theorie (1975) und betrachtet Stereotype als Fragmente des sprachlichen Weltbildes. Er plädiert dabei für eine weite Auffassung des Stereotypbegriffs und definiert ihn als ,subjektiv determinierte mentale Vorstellung eines Objekts, die sowohl dessen beschreibende als auch wertende Merkmale umfasst und Ergebnis der Wirklichkeitsinterpretation im Rahmen der gesellschaftlichen kognitiven Modelle darstellt" (BARTMIŃsKI 1998: 64, Übers.: M.F.). ${ }^{3}$

Den Gutachterinnen dieses Beitrags danke ich für freundliche Anregungen und hilfreiche Hinweise.

Die deutsche Abkürzung SWB stammt aus dem Beitrag von BARTMIŃSKI (2012) und wird auch von mir in diesem Beitrag verwendet.

3 ,,...$]$ subiektywnie determinowane wyobrażenie przedmiotu, obejmujące zarówno cechy opisowe, jak i wartościujące obraz oraz będące rezultatem interpretacji rzeczywistości w ramach społecznych modeli poznawczych". 
Eine solche Definition bedeutet, dass Stereotype als individuell gebildete Vorstellungen und gesellschaftlich gefestigte kognitive Repräsentationseinheiten (Konzepte) betrachtet werden. Die weite Auffassung der Stereotype äußert sich darin, dass diese sich nicht nur auf bestimmte ethnische oder soziale Gruppen beschränken, sondern auch in Bezug auf andere Objekte - Tiere, Pflanzen oder Ereignisse - gebildet werden. Demzufolge kann man von dem Stereotyp des Deutschen, der Mutter, des Fuchses oder von der stereotypen Konzeptualisierung des Kosmos (die auf- und untergehende Sonne) sprechen. Die weite Definition des Stereotyps besteht auch darin, dass zu dieser Gruppe nicht nur negativ wertende Stereotype (Vorurteile), sondern auch positive Stereotype gerechnet werden. Somit verbindet sich das Thema der Stereotypie mit dem anderen in der Ethnolinguistik auch aufgegriffenen Thema der Wertung und deren sprachlichen Indikatoren. BARTMIŃSKI (2007: 133) legt dabei Wert auf die in den Wortbedeutungen enthaltenen, aber nicht obligatorischen, wertenden Merkmale, die er als kulturelle Konnotationen (vgl. Putnam 1975) bezeichnet.

Grundsätzlich für die Rekonstruktion des sprachlichen Weltbildes und für die Analyse der Bedeutung von Stereotypen ist in diesem Ansatz die Methode der Kognitiven Definition, die zum ersten Mal in BARTMIŃsKis „Wörterbuch der sprachlichen Volksstereotype. Probeheft“ (Stownik ludowych stereotypów językowych, Zeszyt próbny, 1980) ihre Anwendung fand, sowie die Begriffe der Profilierung und des Profils als Resultat des genannten Prozesses. Im Gegensatz zu der lexikographischen Definition beschränkt sich die kognitive Definition nicht nur auf systembezogene, notwendige und ausreichende Merkmale der Bedeutung, sondern sie berücksichtigt alle aus der Konzeptualisierung resultierenden kategorialen, charakteristischen und evaluierenden Merkmale des Objekts und dessen inhaltliche Struktur.

Das Basiskonzept, die stereotype Vorstellung eines Objekts und dessen Struktur unterliegt auf der Textebene einer intentionalen Profilierung des Subjekts, die in der Ethnolinguistik viel weiter verstanden wird als in der kognitiven Grammatik von LANGACKER $(2005,2009)$. Es handelt sich dabei um

„eine subjektive (d.h. vom Subjekt durchgeführte) kognitiv-sprachliche Operation, die in der Gestaltung der Objektkonzeptualisierung nach solchen Aspekten (Subkategorien, Facetten) wie Herkunft, Merkmale, Aussehen, Funktionen, Ereignisse und Erlebnisse besteht und im Rahmen eines bestimmten Wissenstyps sowie gemäß einem bestimmten Standpunkt durchgeführt wird." (BARTMIŃsKi 2007: 99, Übers.: M.F.) ${ }^{4}$

Die Profilbildung bedeutet also die Hervorhebung bestimmter Merkmale je nach dem Kontext, wobei es sich nicht um mehrere unterschiedliche Bedeutungen handelt, sondern um die unterschiedliche Art der Wissensorganisation im Rahmen derselben Grundbedeutung. Infolge der Profilierung werden manche Aspekte salienter als andere (vgl. Zinken 2004: 116-117). Die Profilbildung erfolgt also nicht nur in Bezug auf die konzeptuelle Basis des Objekts wie bei LANGACKER (2009), sondern auch in Bezug auf den ,Erfahrungsrahmen' („rama

4, ,[...] profilowanie jest subiektywną (tj. mającą swój podmiot) operacją językowo-pojęciową polegającą na swoistym kształtowaniu obrazu przedmiotu poprzez ujęcie go w określonych aspektach (podkategoriach, fasetach), takich jak np. pochodzenie, cechy, wygląd, funkcje, zdarzenia, przeżycia itp., w ramach pewnego typu wiedzy i zgodnie z wymogami określonego punktu widzenia". 
doświadczeniowa“), d.h. auf den ganzen kulturellen Hintergrund des Begriffs (vgl. „frames“, FILLMORE 1985), auf alle visuell wahrnehmbaren und im Erkenntnisakt konzeptualisierten Elemente sowie auf alles, was kulturell gefestigt wird und so zum Bestandteil des kollektiven Wissens wird. Die Profilbildung ist auch von solchen subjektorientierten Faktoren wie Kategorisierung, Gesichtspunkt und Perspektive sowie Rationalitätstyp und Wertesystem abhängig.

Die entscheidende Rolle spielt dabei die Kategorie des Gesichtspunktes und der Perspektive, die nicht nur als Betrachtungsparameter (LANGACKER 2005, 2009), sondern auch als kulturell geprägte Faktoren, d.h. als Standpunkt und als Interpretationsperspektive aufgefasst werden. BARTMIŃSKI (2007: 120-121) weist dabei auf die Kategorie des kollektiven Subjekts und des kollektiven Standpunktes hin, die durch typisierende Ausdrücke wie von dem deutschen Standpunkt aus signalisiert werden. Die einzelnen Stereotype können also über mehrere textuelle Profile verfügen, die von dem Basisprofil abgeleitet werden und auf diese Weise ein konzeptuelles Netz bilden können.

Bei nationalen Stereotypen rücken im Vergleich zu anderen Stereotypen besonders solche Merkmale in den Fokus, die mit dem Aussehen, mit der Sprache, mit Essgewohnheiten (Deutsche als Biertrinker, Franzosen als Froschfresser, Italiener als Spaghettifresser), Charaktereigenschaften sowie mit nationalen Vorlieben und Lieblingsfreizeitbeschäftigungen (Russen als Harmonikaspieler) und mit Kleidung verbunden sind (vgl. BARTMIŃSKI 1998: 108-109).

Nationale Stereotype als Fragmente des sprachlichen Weltbildes stützen sich auf die elementare Dichotomie ,eigen vs. fremd', die es ermöglicht, zwischen der Selbst- und Fremdwahrnehmung, also zwischen den Auto- und Heterostereotypen zu unterscheiden.

Die Lubliner Schule der Ethnolinguistik macht auch auf die Dynamik von Stereotypen aufmerksam. Das Profil des Stereotyps kann sich ändern und ist jeweils vom Situationskontext, vom Standpunkt des Konzeptualisators sowie von dessen axiologischen Annahmen abhängig. BARTMIŃSKi (1998: 256) illustriert diesen Prozess mit der diachronischen Analyse des sich wandelnden Stereotyps des Deutschen im polnischen Weltbild (vom 16. bis zum 20. Jahrhundert) und unterscheidet anhand des sprachlichen Materials folgende drei Stereotypenprofile dieser Zeit:

a) das Stereotyp des Deutschen als eines Fremden, das sich schon in der Nationalitätsbezeichnung Niemiec manifestiert, die laut der Volksetymologie aus dem Adjektiv niemy (,stumm') abgeleitet wird (16./17. Jahrhundert),

b) das Stereotyp des feindlichen Deutschen, das seinen Ausdruck in solchen Sprichwörtern wie Jak świat światem, nigdy Niemiec nie będzie Polakowi bratem / (,Solange die Welt besteht, wird der Deutsche dem Polen nie Bruder sein') oder die Kollokation buta niemiecka (,deutscher Hochmut') findet (19. Jahrhundert und die erste Hälfte des 20. Jahrhunderts), und

c) ein positives Bild des Deutschen als eines offenen Europäers (Ende des 20. Jahrhunderts).

Der Stereotypenwandel betrifft sowohl die beschreibende als auch die wertende Komponente. Die Änderung der axiologischen Orientierung, die oft erst in längerer zeitlicher Perspektive zu beobachten ist, zieht auch die Veränderungen in der evaluativen Komponente nach sich. Die Dynamik des Stereotyps und dessen Profile sind somit historisch und kulturell bedingt. 
Das sprachliche Weltbild, Stereotype und deren Profile sowie kollektive Standpunkte können in dieser Forschungskonzeption anhand von drei Typen sprachlicher Daten, d.h. von system-, text- und auch von konventionsbezogenen Daten (Umfragen), rekonstruiert werden.

\section{Das Stereotyp des Polen im deutschen Weltbild des 19. Jahrhunderts - eine ethnolinguistische Studie}

Das Ziel meiner Analyse ist es, im Rahmen der präsentierten Forschungskonzeption und Methodologie die Bedeutung der Heterostereotype über Polen im 19. Jahrhundert zu rekonstruieren. Es entsteht dabei die Frage, welche Stereotype für diese Zeitperiode charakteristisch sind, ob und wie sich das Polenbild im 19. Jahrhunderts verändert und mit welchen Mitteln es ausgedrückt wird. Für die Zwecke meiner Analyse stütze ich mich auf Phraseologismen und Metaphern, die zwei lexikographischen Quellen sowie dem DWDS Korpus (Textarchiv) entnommen wurden.

\subsection{Das deutsche Polenbild im DWDS Wörterbuch und im Lexikon OldPhras}

Das im Kontext des polnischen Deutschenbildes angeführte Stereotyp des Fremden und die Dichotomie ,eigen vs. fremd' werden in den deutschen Nationalitätsbezeichnungen nicht exponiert. Der deutsche Eigenname der Pole (DWDS) verweist auf die ethnische Zugehörigkeit. Neben diesem Eigennamen funktionieren im Deutschen auch solche Bezeichnungen wie der Pollacke, die Pollackei (DWDS), die etymologisch auf die polnische Nationalitätsbezeichnung zurückgehen und mit einer negativen Bewertung verwendet werden.

Das phraseologisch gefestigte Polenbild weist positive und negative Merkmale auf. Zu den positiven Merkmalen, die sich phraseologisch manifestieren, gehört der Mut und die Tapferkeit der polnischen Nation, die in der Redewendung Noch ist Polen nicht verloren "noch ist die Sache nicht gescheitert, noch gibt es den Grund zur Hoffnung" (OLdPhras) vorkommt.

Einen starken Einfluss auf die deutsche Gesellschaft hatte aber vor allem das Stereotyp des unordentlichen Polen, das seinen Ausdruck am deutlichsten in der gut bekannten Metapher polnische Wirtschaft (OldPhras) und in den mit ihr verbundenen Phraseologismen findet. Diese häufig verwendete Metapher lässt sich in ihren Ursprüngen auf eine Metonymie zurückführen, die für all das stand, was mit Polen als Land und als Nation assoziiert wurde. Sie profiliert solche Eigenschaften wie Unordentlichkeit und Unwirtschaftlichkeit von Polen. Die Bedeutung dieser lexikalischen Einheit korrespondiert mit solchen politischen Ereignissen in Polen, wie der Verfall der Adelsrepublik und der Untergang der Staatlichkeit. Sie bildet auch den Ausgangspunkt für weitere Metaphern wie z.B. der polnische Sejm, derpolnische Reichstag,

5 Alle Korpusbelege stammen aus dem Deutschen Textarchiv (1800-1900), https://www.dwds.de. Dabei waren die quantitative Analyse des gesammelten Korpusmaterials sowie die Bestimmung des Vorkommens von einzelnen Metaphern aus technischen Gründen nicht möglich. Die statistischen Auswertungen sind im DWDS Korpus (Textarchiv) erst ab 1945 zugänglich. 
die auch in derselben Bedeutung verwendet werden (vgl. Czachur 2015). Eine ähnliche Bedeutung weist auch die deutsche Redewendung da (heute) ist Polen offen (OldPhras) auf, die von der Metapher polnische Wirtschaft abgeleitet wurde und auf großes Durcheinander und militärische Schwäche des Landes (durch Aufstände verursacht) verweist.

\subsection{Das deutsche Polenbild in Texten des deutschen Textarchivs (1800-1900)}

Den zweiten Teil der Analyse stellt die Rekonstruktion von stereotypen Konzeptualisierungen von Polen am Beispiel metaphorischer Äußerungen in Texten dar, die im Vergleich mit den Phraseologismen über ein großes Bild- und Emotionspotenzial verfügen. Vom Standpunkt der Demokraten und Liberalen aus wird Polen einerseits als tapfere und heldenmütige Nation, andererseits als Opfer der Expansionspolitik der europäischen Großmächte konzeptualisiert.

1. Sodann erhob er die Tapferkeit und die Kriegszucht der Pohlen, von der ich selbst Beweise hatte, und an welcher ich also nicht zweifelte. (Seume, Johann Gottfried (1803): Spaziergang nach Syrakus im Jahre 1802. Braunschweig.)

2. Die Polen sterben den schönen Heldentodt, oder sie leben für die Freiheit. (Börne, Ludwig (1832): Briefe aus Paris Bd. 2. Hamburg.)

3. Hinter beiden der würgende Czar, der, noch bluttriefend vom Morde des heldenmüthigen Polens, aller Freiheit und Civilisation den Herzstoß versetzen will; von diesem dreifachen Feinde sehen wir das Vaterland bedroht. (Wirth, Johann Georg August (1832): Das Nationalfest der Deutschen zu Hambach. Heft 1. Neustadt.)

4. In Deutschlands starken Eichen, im schauerlichen Rauschen, die göttliche Nähe verkündend, mahnt er mächtig zur Thatkraft, er deutet auf Warschau's rauchende Thürme, auf die Wunden des unglücklichen Polens, deutet auf das russische Unthier, das den blutigen Blick auf Deutschland richtet. (Wirth, Johann Georg August (1832): Das Nationalfest der Deutschen zu Hambach. Heft 1. Neustadt.)

Die ersten zwei Belege signalisieren explizit die genannten Merkmale durch generalisierende Aussagen und verweisen somit auf die eher positiv bewertete Konzeptualisierung von Polen. Die Metaphern in Belegen (3-4) signalisieren dagegen eine konträre Konzeptualisierung von Polen als einem leidenden Volk und zugleich dessen Opferbereitschaft für andere Nationen. Sie konzentrieren sich vor allem auf die russische Politik gegen Polen und Deutschland. In der ersten Metapher wird die aggressive Polenpolitik Russlands als Mord Polens durch das ihr zugrundeliegende metaphorische Muster Die Teilungen Polens sind Mord DeR NATION ${ }^{6}$ wiedergegeben und global als Herzstoß für die Zivilisation und Europa signalisiert. Diese aggressive Politik Russlands wird auch durch zwei weitere Metaphern im Beleg (4) gefestigt, in denen Polen als blutender Organismus und Russland als Ungeheuer, z.B. die Wunden Polens, das Unthier, das den blutigen Blick aufDeutschland richtet dargestellt werden.

Alle Metaphern in den Belegen (3) und (4) werden von negativ bewertenden Bezeichnungen Russlands wie der würgende Czar, bluttriefend, den blutigen Blick richten begleitet und weisen dadurch eine persuasive und emotionalisierende Wirkung auf den Rezipienten auf.

6 Zur Hervorhebung der kognitiven Auffassung der Metapher und Markierung der metaphorischen Konzepte werden in der Analyse KAPITÄLCHEN verwendet. 
In der zweiten Gruppe von Belegen werden das stereotype Polenbild und die politische Lage des Landes im 19. Jahrhundert mit Hilfe der Todesmetaphorik wiedergegeben, z.B.:

5. Jordan habe das unpassende, das schauerliche Wort ausgesprochen, Polen war nach der ersten Theilung schon eine Leiche, dann seien die Könige, die es noch einmal getheilt, Leichenzerreißer, Hyänen, und die Nationalversammlung sei der Todtengräber (Moritz Arndt und die Rechte lärmen.) (Neue Rheinische Zeitung Nr. 58. Köln, 28. Juli 1848.)

6. Wäre Polen todt, wir müßten es auferwecken. (Neue Rheinische Zeitung Nr. 58. Köln, 28. Juli 1848.)

7. Polen ist vorläufig zu Grabe gegangen ebenso sehr an seiner inländischen Aristokratie als an den Einbrüchen der Ausländer; es ist so zusagen die Personificirung des dreieinigen Prinzips welches einst die Welt beherrschen wird: Freiheit, Gleichheit, Brüderlichkeit. (Neue Rheinische Zeitung Nr. 202. Köln, 22. Januar 1849. Beilage.)

8. Der Untergang Polens ist ein furchtbares Beispiel einer solchen Zerreißung und Tödtung eines Stats. (Bluntschli, Johann Caspar (1868): Das moderne Völkerrecht der civilisirten Staten. Nördlingen.)

In Belegen (5) - (8) dominiert die Metapher Teilung Polens ist Tod des staates. ${ }^{7}$ Das metaphorische Bild wird durch weitere Analogien zwischen Domänen präzisiert, d.h. die Könige werden als Leichenzerreißer, die Nationalversammlung als Todtengräber dargestellt. Dieselbe Metapher wird auf der Textebene mit unterschiedlichen Sprachmitteln ausgedrückt, d.h. sowohl lexikalisch als ausgebaute Nominalphrasen (Polen als Leiche, Untergang Polens, Tödtung eines Stats) als auch verbal als Satzkonstruktionen (z.B. Wäre Polen todt, wir müssten es auferwecken und Polen ist vorläufig zu Grabe gegangen). Die Todesmetapher in (6) impliziert auch die Anwesenheit einer anderen daraus resultierenden Metapher: Rettung Polens ist Auferweckung Der Nation. Die Nominalphrase der Untergang Polens kann übrigens auch als eine Metapher an sich betrachtet werden und ist als Orientierungsmetapher zu klassifizieren, der das metaphorische Muster NACH UNTEN GEHEN IST SCHLIMM / IsT ETWAS Negatives zugrundeliegt. Solche Konzeptualisierungen zeichnen sich auch durch ein starkes Emotionspotenzial aus.

Eine wichtige Stelle in dem gesammelten Material nehmen auch die mit der Todesmetaphorik verbundenen Metaphern religiöser Provenienz ein, wie z.B.:

9. Ja, Polen ist der Pfahl im russischen Fleische; an dem mißhandelten Polen verblutet die russische Despotie; das Kreuz, an welches sie den Märtyrer geschlagen hat, es wird ihr selber zu dem Sünderbalken, an welchem sie ihr scheußliches Leben endet. (Bakunin, Michail Alexandrowitsch (1848): Aufruf an die Slaven. Koethen.)

10. Wir verdammten feierlich jene verbrecherische Politik, welche Polen dreimal zerriß und seine traurigen Reste abermals zerreißen will, und drückten die lebhafte Hoffnung aus, daß die Auferstehung dieses edeln, heiligen Märtyrervolkes bald das Zeichen zur Erlösung unser Aller aus der alten Knechtschaft geben würde. (Bakunin, Michail Alexandrowitsch (1848): Aufruf an die Slaven. Koethen.)

11. Polen ist der Völker-Christus, es hat seinen Kreuzestod vollbracht; wir aber, die Erben und Apostel der gesetzlichen Freiheit, wir sollen vor der Welt und der Zeit durch unser Beispiel Zeugniß geben für die Gültigkeit dessen, was die Vorsehung in der Geschichte unseres Landes geoffenbaret hat. (Neue Rheinische Zeitung Nr. 217. Köln, 9. Februar 1849.)

7 Die Begriffe: ,Nation` und ,Staat` werden hier synonym verwendet. 
12. Auf die Polen läßt sich die Geschichte jener zwei Mütter und des Königs Salomo anwenden; wie jene edle Mutter ihr Kind lieber ganz der Fremden hingeben als theilen lassen wollte, so wollen jetzt die Polensöhne lieber ihre Mutter ganz dem fremden deutschen Volke hinwerfen als zerfetzen lassen. (Neue Rheinische Zeitung Nr. 217. Köln, 9. Februar 1849.)

Im Beleg (9) haben wir es mit einem ganzen Komplex von religiösen Metaphern zu tun. Die Teilungen und das tragische Schicksal Polens werden als Kreuzigung konzeptualisiert, und Polen wird dem biblischen Vergleich nach als Pfahl im russischen Fleische dargestellt, d.h. als etwas körperlich oder seelisch Peinigendes, was einen nicht zur Ruhe kommen lässt. ${ }^{8}$ Die Wiederherstellung Polens wird also als seine Auferstehung konzeptualisiert (s. Beleg 5). Die Metapher Polenteilungen sind Kreuzigung der Nation im Beleg (9) wird auch im Textausschnitt (11) in der Metapher Polen IST VöLKer-Christus fortgesetzt. Der Vergleich mit Christus wird an weiteren Textstellen noch erweitert, indem die deutschen Demokraten sich selbst sehr positiv als die Erben und Aposteln der gesetzlichen Freiheit konzeptualisieren. Dieser direkte Vergleich mit Christus bringt auf der pragmatischen Ebene zusätzliche Implikationen über die besondere Rolle der polnischen Nation in der Geschichte mit sich. Diese Konzeptualisierung von Polen wird auch lexikalisch als einfache Nominalphrase: Märtyrer, Märtyrervolk oder erweitert durch expressive Adjektive wie das misshandelte Polen, das edle heilige Marterland hervorgehoben.

Die letzte Metapher in dieser Gruppe (12) wird auch in den biblischen Kontext eingebunden. Polen wird in Bezug auf die konzeptuelle Domäne der Familienverhältnisse als Mutter und polnische Bürger werden in Analogie als deren Kinder (Polensöhne) (Beleg 11,4) konzeptualisiert. Ein solcher Vergleich impliziert einen besonderen Wert der eigenen Staatlichkeit und verweist auch auf solche Charaktereigenschaften von Polen wie Liebe und Respekt zum Vaterland. Der Korpusbeleg verweist auch explizit auf das Stereotyp des Deutschen als eines Fremden.

Die letzte Gruppe von Belegen ist der im deutschen Polendiskurs gut bekannten Metapher polnische Wirtschaft und den rund um sie funktionierenden Ausdrücken gewidmet.

13. Wenn die Pohlen noch unter einem einzigen Herrn wären, so liesse sich durch eben diese Eifersucht noch Rettung denken. (Seume, Johann Gottfried (1803): Spaziergang nach Syrakus im Jahre 1802. Braunschweig.)

14. Die Ohnmacht, der Mangel an politischer und rechtlicher Haltung, welche wir in Polen wahrnehmen, hat ihren Hauptgrund in der einseitigen Anlage dieses Landes zum Ackerbau, und demnach in einseitiger Ausbildung des Gesetzes. (Müller, Adam Heinrich (1809): Die Elemente der Staatskunst. Bd. 1. Berlin.)

Die hier explizit abgerufene Bedeutung der Metapher polnische Wirtschaft beschränkt sich nicht nur auf das Merkmal „,unvorstellbare Unordnung“", sondern profiliert in diesem Beispiel auch weitere negative Nationaleigenschaften von Polen, wie z.B. Mangel an Einigkeit (wenn die Poblen noch unter einem einzigen Herrn wären) oder auch die Rückständigkeit (einseitige

8 Nach 2. Korinther 12, 7, in: Duden Online Wörterbuch, www.duden.de (28.07.2018). 
Anlage zum Ackerbau). Auf diese Weise trägt sie wesentlich zur Konstituierung und sogar zur Erweiterung und Festigung des negativ bewerteten Stereotyps des unordentlichen Polen bei.

In zwei weiteren Belegen dagegen dient die lexikalisierte Metapher polnische Wirtschaft nicht nur als Kern der stereotypen Konzeptualisierung von Polen, sondern auch als Bezugspunkt für die Konfrontation mit anderen nationalen Stereotypen, wie in folgenden Belegen:

15. Die beiden Nationen haßten sich nicht nur, sie verachteten einander auch; wie der Deutsche alle Niedertracht und Unredlichkeit mit dem Worte „polnische Wirthschaft“ bezeichnete, so konnte sich der Pole den sparsamen Ordnungssinn der Deutschen nur aus einem angeborenen Bedientengeiste erklären. (Treitschke, Heinrich von (1889): Deutsche Geschichte im neunzehnten Jahrbundert. Bd. 4: Bis zum Tode König Friedrich Wilhelms III. Leipzig.)

16. Ziemlich verschiedenartig stehen sich noch heute der extravagante, verschwenderische Pole, der nach dem Sprichwort auf der Jagd einen Hahn erlegt, um beim Essen einen Ochsen zu verspeisen, der, stets elastisch begeistert, heiter und nachlässig „polnische Wirtschaft“ treibt, dann der ackerbauende, stabile, altväterische, um das heilige Kiew sich gruppierende, sentimentale, liederreiche Kleinrusse und endlich der moderne, mit Mongolen- und Tatarenblut viel mehr gemischte, dem Handel und dem Gewerbe viel mehr zugeneigte, seit dem 16. Jahrhundert zur Herrschaft gelangte Großrusse gegenüber. (Schmoller, Gustav (1900): Grundriß der Allgemeinen Volkswirtschaftslehre. Bd. 1. Leipzig)

Im Beleg (15) werden die Stereotype des Polen und des Deutschen miteinander konfrontiert. Die mit der Metapher polnische Wirtschaft ${ }^{9}$ assoziierten Eigenschaften Niedertracht und Unredlichkeit werden mit dem ordnungsliebenden und sparsamen Deutschenbild kontrastiert.

Das negative Polenbild wird durch die unterstellte Bewertung der Deutschen als Bediener verstärkt, was auf solche Eigenschaften wie Respektlosigkeit und Hochmut verweisen kann. Der stereotype Pole fällt auch negativ im Vergleich mit anderen slawischen Nationen aus. Er wird als extravaganter, verschwenderischer, fauler und leichtsinniger Mensch konzeptualisiert, im Gegensatz zu Klein- und Großrussen, denen positive Züge zugeschrieben werden. Die negative Bewertung äußert sich in diesem Falle nicht nur in dem Sprichwort der Pole erlegt einen Habn, um beim Essen einen Ochsen zu verspeisen [...], sondern auch in den unkonventionellen, antonymen Wortverbindungen wie stets elastisch begeistert, heiter und nachlässigpolnische Wirtschaft treiben, was auf der semantischen Ebene des Textes als Zeichen der Ironie verstanden werden kann.

\section{Fazit}

Die Anwendung der im Rahmen der Lubliner Schule der Ethnolinguistik erarbeiteten Methode der kognitiven Definition sowie die Arbeit mit unterschiedlichen Typen von sprachlichen Daten eröffnet die Möglichkeit, die stereotypen und vielfältigen Konzeptualisierungen von Polen als Land und Nation im 19. Jahrhundert möglichst vollständig zu rekonstruieren.

Die Analyse des Polenbildes anhand von lexikographischen Daten lässt zwei Basisstereotype im deutschen Weltbild rekonstruieren, d.h. das Stereotyp eines heldenmütigen Polen und

9 Mehr zur Metapher polnische Wirtschaft vgl. OrŁowsKi 2003, KochanOwSKA-NiEBORAK 2007, Czachur 2015. 
das schon früher gefestigte Stereotyp eines schlecht funktionierenden Landes. Die Analyse des Polenbildes anhand von textbezogenen Daten aus dem Korpus ermöglicht es, ein differenzierteres Polenbild zu rekonstruieren und folgende Stereotypenprofile zu erkennen:

a) Von dem Stereotyp eines heldenmütigen Polens werden die Stereotypenprofile einer toten, ihrer Staatlichkeit beraubten Nation sowie das Profil eines Märtyrervolkes und das Profil einer sich für ihre Kinder aufopfernden Mutter abgeleitet. Diese Stereotypenprofile, oft durch Metaphern religiöser Provenienz ausgedrückt, werden positiv bewertet und sind besonders in den Texten aus der ersten Hälfte des 19. Jahrhunderts zu finden.

b) Das zweite in der Metapher polnische Wirtschaft und mittels bestimmter Phraseologismen gefestigte Stereotyp einer unwirtschaftlichen Nation und eines schlecht funktionierenden Landes wird anhand von textbezogenen Korpusdaten noch um solche Merkmale wie Rückständigkeit, Leichtsinn, Verschwendung, Extravaganz und Respektlosigkeit für andere Nationen erweitert. Das negativ bewertete Stereotyp kommt in Texten aus der zweiten Hälfte des 19. Jahrhunderts vor. Die durchgeführte Analyse lässt somit auf eine Dynamik des Polenbildes im 19. Jahrhundert schließen. Der Stereotypenwandel betrifft nicht nur die inhaltliche, sondern auch die emotionale Ebene, das positiv bewertete Stereotyp der kämpfenden Polen wird durch ein negatives Bild ersetzt. Die Dynamik des Polenbildes und dessen Profile sind in diesem Falle nicht kulturell, sondern historisch bedingt.

Das DWDS Korpus ermöglicht die Arbeit mit einem weiten Spektrum von differenzierten Textgattungen, die aus unterschiedlichen Zeitperioden des 19. Jahrhundert stammen, und erlaubt somit den allmählichen Wandel von Stereotypen zu erfassen und zu beschreiben.

\section{Bibliographie}

\section{Quellen}

Duden Online-Wörterbuch, URL: https://www.duden.de (8.08.2018).

DWDS-Wörterbuch $=$ Das Digitale Wörterbuch der deutschen Sprache, https://www.dwds.de (8.08.2018).

DWDS Korpus - (Deutsches Textarchiv (1800-1900)), https://www.dwds.de (8.08.2018).

OldPhras = Online-Lexikon zur diachronen Phraseologie, https://www.oldphras.net (8.08.2018).

\section{Sekundärliteratur}

ApresJAN, Jurij (1994): Naiwny obraz świata a leksykografia [Das naive Weltbild und Lexikographie]. In: Etnolingwistyka 6, 5-12.

BARTMIŃs KI, Jerzy (2007): Językowe podstawy obrazu świata [Die sprachlichen Grundlagen des Weltbildes]. Lublin: Wydawnictwo UMSC.

BARTMIŃSKI, Jerzy (1998): Stereotypy mieszkają węzyku. Studia etnolingwistyczne [Stereotype wohnen in der Sprache. Ethnolinguistische Studien]. Lublin: Wydawnictwo UMSC. 
BARTMIŃski, Jerzy (1980): Stownik ludowych stereotypów jezykowych. Zeszytpróbny [Wörterbuch der sprachlichen Volksstereotype. Probeheft]. Wrocław: Wydawnictwo Uniwersytetu Wrocławskiego.

BartmiŃsKi, Jerzy (2012): Der Begriff des sprachlichen Weltbildes und die Methoden seiner Operationalisierung. Aus dem Polnischen von CZAChur, Waldemar / Schramm, Wolfgang. In: Tekst i dyskurs - Text und Diskurs 5/2012, 269-289 (http://tekst-dyskurs.eu/images/pdf/zeszyt_5/ bartminski.pdf, 5.08.2018).

Busse, Dietrich (2012): Frame-Semantik: ein Kompendium. Berlin; New York: de Gruyter.

Czachur, Waldemar (2015): Semantischer und funktionaler Wandel von Metapher und Metonymie. Polnische Wirtschaft im deutschen Mediendiskurs zur Wirtschaftskrise. In: Spiess, Constanze / KöpcKe, Klaus-Michael (Hg.): Metapher und Metonymie. Theoretische, methodische und empirische Zugänge (= Empirische Linguistik 1). Berlin; New York: de Gruyter, 227-246.

Czachur, Waldemar (2016): Kultursensitive Linguistik - eine Spezialität der fremdsprachigen Germanistik? In: Duś, Magdalena / KoŁodziej, Robert / Rojek, Tomasz (Hg.): Wort - Text - Diskurs (= Danziger Beiträge zur Germanistik 53). Frankfurt a.M.: Peter Lang, 337-349.

Fillmore, Charles John (1975): Abstracts. Proceedings of the First Annual Meeting of the Berkeley Linguistics Society (1975), 123-131.

Fillmore, Charles John (1985): Frames and the semantics of understanding. In: Quaderini di Semantica vol. 6, no. 2, 222-254.

HuмвоLDT, Wilhelm von (1836): Über die Kawi-Sprache auf der Insel Java, nebst einer Einleitung über die Verschiedenheit des menschlichen Sprachbaues und ihren Einfluss auf die geistige Entwickelung des Menschengeschlechts. Berlin: Königliche Akademie der Wissenschaften.

Kochanowska-Nieborak, Anna (2007): Francuzi Pótnocy. Obraz Polski i Polaków w niemieckich leksykonach konwersacyjnych XIX wieku [Das Polenbild in deutschen Konversationslexika des 19. Jahrhunderts] (= Zrozumieć Niemcy 6). Wrocław: ATUT.

LANGACKer, Ronald W. (1987): Foundations of Cognitive Grammar. Theoretical Prerequisites vol. 1. Stanford: Stanford University.

LANGACKER, Ronald W. (2005): Wyktady z gramatyki kognitywnej [Vorlesungen in der kognitiven Grammatik]. Lublin: Wydawnictwo UMSC.

LANGACKer, Ronald W. (2009): Gramatyka kognitywna. Wprowadzenie [Die kognitive Grammatik. Eine Einführung]. Kraków: Universitas.

Lippmann, Walter (1922): Public Opinion. New York: Harcourt Brace.

OrŁowski, Hubert (2003): Zur Lesbarkeit von Stereotypen: der deutsche Polendiskurs im Blick historischer Stereotypenforschung und historischer Semantik (= Beihefte zum Orbis Linguarum 31). Wroclaw: ATUT.

Putnam, Hilary (1975): The Meaning of Meaning. Mind, Language and Reality. Cambridge: Cambridge University Press, 215-271.

Stefanowitsch, Anatol (2006): Introduction. In: Ders. / Gries, Stefan (Hg.): Corpus-Based Approaches to Metaphor and Metonymy. Berlin; New York: Walter de Gruyter, 1-16.

WeISGERBER, Leo (1971): Die geistige Seite der Sprache und ihre Erforschung (= Sprache der Gegenwart 15). Düsseldorf: Pädagogischer Verlag Schwann.

ZINKEN, Jörg (2004): Metaphors, stereotypes, and the linguistic picture of the world: impulses from the Ethnolinguistic School of Lublin. In: metaphorik.de 7, 115-136. 